\title{
The Spectrometer Telescope for Imaging X-rays (STIX) on board the Solar Orbiter mission
}

A. O. Benz ${ }^{\text {a }}$, S. Krucker ${ }^{\text {ar }}$, G. J. Hurford ${ }^{\text {ar }}$, N. G. Arnold ${ }^{\mathrm{a}}$, P. Orleański ${ }^{\text {af }}$, H.-P. Gröbelbauer ${ }^{\mathrm{a}}$, S. Kobler $^{\mathrm{a}}$, L. Iseli ${ }^{\mathrm{a}}$, H. J. Wiehl ${ }^{\mathrm{a}}$, A. Csillaghy ${ }^{\mathrm{a}}$, L. Etesi ${ }^{\mathrm{a}}$, N. Hochmuth $^{\mathrm{a}}$, M. Battaglia ${ }^{\mathrm{a}}$, M. Bednarzik ${ }^{\mathrm{b}}$,

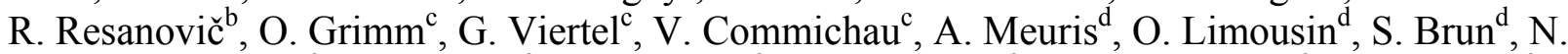

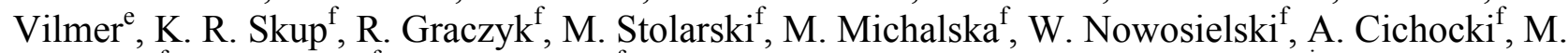

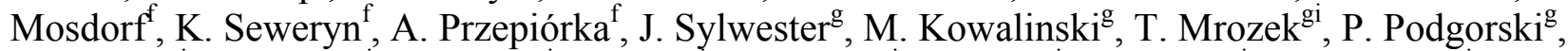

G. Mann ${ }^{\mathrm{i}}$, H. Aurass ${ }^{\mathrm{i}}$, E. Popow ${ }^{\mathrm{i}}$, H. Önel, $\mathrm{i}^{\mathrm{i}}$ F. Dionies ${ }^{\mathrm{i}}$, S. Bauer ${ }^{\mathrm{i}}$, J. Rendtel ${ }^{\mathrm{i}}$, A. Warmuth ${ }^{\mathrm{i}}$, M.

Woche $^{i}$, D. Plüschke ${ }^{i}$, W. Bittner, J. Paschke, D. Wolter ${ }^{i}$, H. F. Van Beek ${ }^{\mathrm{j}}$; F. Farnik ${ }^{\mathrm{k}}$, J.

Kasparova $^{\mathrm{k}}$, A. M. Veronig ${ }^{1}$, I. W. Kienreich ${ }^{1}$, P. T. Gallagher ${ }^{\mathrm{m}}$, D. S. Bloomfield ${ }^{\mathrm{m}}$, M. Piana ${ }^{\mathrm{n}}$, A. M. Massone $^{\text {o }}$ B. R. Dennis ${ }^{\mathrm{p}}$, R. A. Schwartz ${ }^{\mathrm{pq}}$, R. P. Lin ${ }^{\mathrm{rs}}$

${ }^{a}$ University of Applied Sciences and Arts Northwestern Switzerland, Windisch, Switzerland; ${ }^{b}$ Laboratory for Micro- and Nanotechnology, Paul Scherrer Institute, Villigen, Switzerland, ${ }^{\mathrm{c}}$ ETH Zürich, Switzerland; ${ }^{\mathrm{d} C E A}$ Saclay, France; ${ }^{\mathrm{e}}$ LESIA, France; ${ }^{\mathrm{f}}$ Space Research Center of Polish Academy of Sciences, Warsaw, Poland; ${ }^{\mathrm{g}}$ Space Research Centre, Polish Academy of Sciences, Wroclaw, Poland; ${ }^{\mathrm{h}}$ Astronomical Institute, University of Poland, Wroclaw, Poland, ${ }^{\mathrm{i}}$ Leibniz-Institut für Astrophysik Potsdam, Potsdam, Germany; ${ }^{\mathrm{j} H}$ H.F. Van Beek Consultancy B.V., Netherlands; ${ }^{\mathrm{k}}$ Astronomical Institute AS CR, Ondrejov, Czech Republic; 'Institute of Physics, University of Graz, Graz, Austria; ${ }^{\mathrm{m}}$ Trinity College Dublin, Ireland; ${ }^{\mathrm{n}}$ Dipartimento di Matematica, Universita di Genova, Genova, Italy; ${ }^{\circ} \mathrm{CNR}$-SPIN Genova, Italy; ${ }^{\mathrm{p}} \mathrm{NASA}$ Goddard Space Flight Center, USA; ${ }^{\mathrm{q}} \mathrm{Catholic}$ University of America, USA; ${ }^{\mathrm{r}}$ Space Sciences Laboratory, UC Berkeley, USA; ${ }^{\mathrm{S}} \mathrm{School}$ of Space Research, Kyung Hee University, Yongin, Korea.

\begin{abstract}
The Spectrometer Telescope for Imaging X-rays (STIX) is one of 10 instruments on board Solar Orbiter, a confirmed Mclass mission of the European Space Agency (ESA) within the Cosmic Vision program scheduled to be launched in 2017. STIX applies a Fourier-imaging technique using a set of tungsten grids (at pitches from 0.038 to $1 \mathrm{~mm}$ ) in front of 32 pixelized CdTe detectors to provide imaging spectroscopy of solar thermal and non-thermal hard X-ray emissions from 4 to $150 \mathrm{keV}$. The status of the instrument reviewed in this paper is based on the design that passed the Preliminary Design Review (PDR) in early 2012. Particular emphasis is given to the first light of the detector system called CalisteSO.
\end{abstract}

Keywords: X-ray imaging, X-ray spectrometer, Solar Physics

\section{INTRODUCTION}

The Spectrometer Telescope for Imaging X-rays (STIX) has been selected to be one of the 10 instruments on ESA's Solar Orbiter mission. Solar Orbiter is a confirmed mission within ESA's Cosmic Vision plan to be launched in 2017. In an elliptical orbit that extends almost to one quarter of the Earth's distance from the Sun, Solar Orbiter will explore the Sun-Heliosphere connection by combining remote sensing imaging spectroscopy observations with in-situ observations of fields and particles. STIX provides imaging spectroscopy of solar X-ray emissions from $\sim 4$ to $150 \mathrm{keV}$, with good spectral resolution and unprecedented spatial resolution and sensitivity (near perihelion). STIX is a European collaboration between Poland, France, Germany, Czech Republic, Austria, and Ireland, with Switzerland as the lead.

Space Telescopes and Instrumentation 2012: Ultraviolet to Gamma Ray,

edited by Tadayuki Takahashi, Stephen S. Murray, Jan-Willem A. den Herder,

Proc. of SPIE Vol. 8443, 84433L · () 2012 SPIE · CCC code: 0277-786/12/\$18 - doi: 10.1117/12.927793 
STIX is based on a Fourier-transform imaging technique similar to that used successfully by the Hard X-ray Telescope (HXT) on the Japanese Yohkoh mission ${ }^{1}$, and related to that used for the Reuven Ramaty High Energy Solar Spectroscopic Imager (RHESSI) mission ${ }^{2}$. A set of 32 cadmium telluride (CdTe) X-ray detectors provide spectral resolution of $1 \mathrm{keV}$ at $6 \mathrm{keV}$. In front of each detector, a pair of tungsten grids with variable pitch is mounted. The transmission through the grid pairs to the detectors is a very sensitive function of the direction of incidence of the X-ray flux. The relative count rates of the detector pixels behind the different sets of grids encode the spatial information that can be subsequently decoded on the ground to reconstruct images of the source region at different X-ray energies (a detailed description of the imaging technique is given in [3]).

The most prominent emission mechanism producing solar hard X-rays is bremsstrahlung of electrons as they encounter ambient ions. For thermal electrons that have a Maxwellian distribution function, hard X-ray emission is typically seen from coronal magnetic loops with temperatures above $\sim 10 \mathrm{MK}$. A typical thermal hard X-ray spectrum is given in Figure 1 showing the thermal bremsstrahlung emission in red. The second component seen at higher energies than the thermal emission is bremsstrahlung emission from electrons with a distribution function different from a Maxwellian. This component is often best represented by a broken power law distribution and is generally referred to as the non-thermal component. The non-thermal emission is produced by electrons that are accelerated by the release of magnetic energy during solar flares. From the spectral observations such as shown in Figure 1, the total energy in accelerated electrons can be estimated revealing that particle acceleration is very efficient with a large fraction (generally $>10 \%$ ) of the total released energy is going into accelerating electrons. While the acceleration site is thought to be in the solar corona, the most intense non-thermal emission is produced when flare-accelerated electrons slam into the much denser chromosphere and produce intense hard X-ray sources at the footpoints of flaring loops (Figure 1, right). While the energy radiated in hard X-ray is negligible, a large part of the deposited energy is radiated away at optical wavelengths. Some of the deposited energy is going into heating chromospheric plasma that then 'evaporates' and fills up the flare loop.

In summary, solar flare hard X-ray observations give quantitative measurements of energy release processes in solar flares. The remote-sensing X-ray measurements made with STIX will determine the intensity, spectrum, timing, and location of accelerated electrons near the Sun. Simultaneously, the size, density, temperature, and energy content of the thermal flare loops are determined. By using this set of diagnostics, STIX plays an important role in enabling Solar Orbiter to achieve two of its major science goals of (1) understanding the acceleration of electrons at the Sun and their transport into interplanetary space and (2) determining the magnetic connection of the Solar Orbiter back to the Sun. In this way, STIX provides an important link between the remote and in-situ instruments of the Solar Orbiter mission.
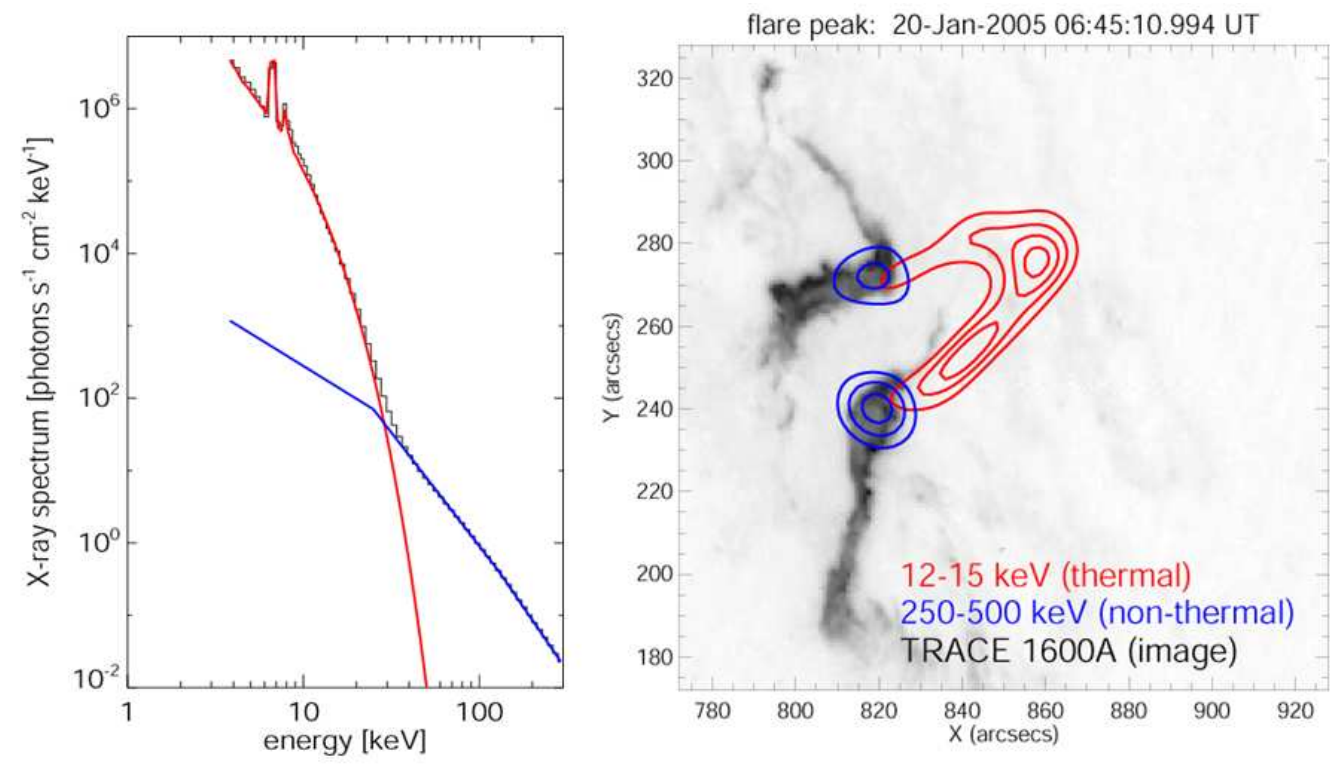

Figure 1.Typical hard X-ray observations of a solar flare (observations are taken by RHESSI). Left: Solar flare spectrum (black histogram) with a thermal (red) and non-thermal (blue) fit to the data. Right: Imaging observations of the same event. The non-thermal emission is seen from the chromospheric footpoints (blue) of the thermal flare loop (red). 


\section{INSTRUMENT}

The STIX instrument consists of three main parts (Figure 2):

1. An X-ray transparent window in the heat shield,

2. An imager with 32 subcollimators, and

3. A spectrometer with 32 cadmium telluride (CdTe) X-ray detectors, one behind each subcollimator.

The instrument performance is summarized in Table 1. The individual subsystems are briefly described in the following sections.
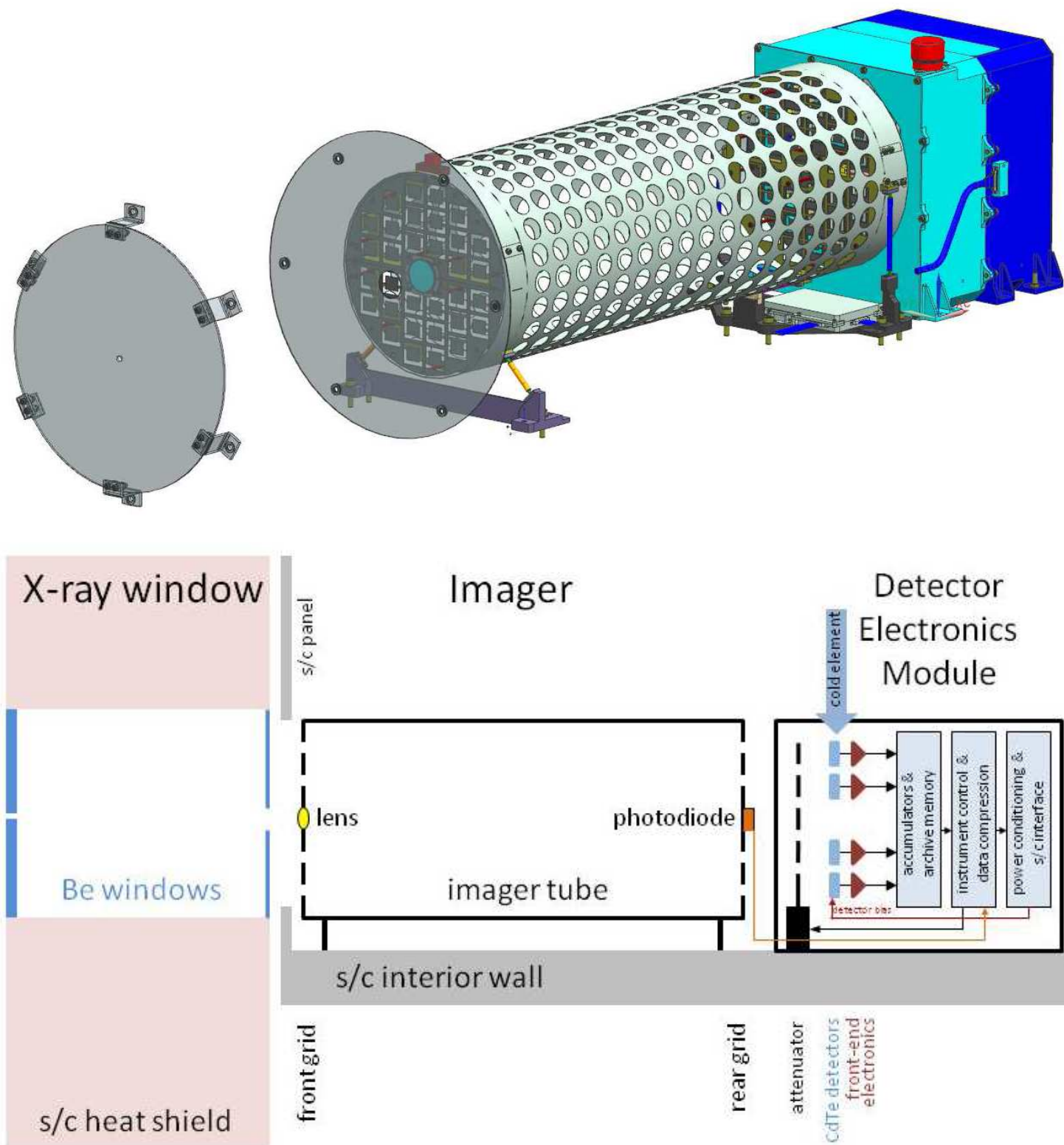

s/c interior wall

Figure 2.Top: The STIX instrument is made up of three mechanically-separate sections: the X-ray windows; the Imager with widely separated grids and aspect system; and the Detector/Electronics Module containing CdTe detectors and electronics. Bottom: Functional block diagram of the STIX instrument. 
Table 1. Instrument performance

$\begin{array}{ll}\text { Energy Range } & 4-150 \mathrm{keV} \\ \text { Energy Resolution (FWHM) } & 1-15 \mathrm{keV} \text { (energy dependent) } \\ \text { Effective area } & 6 \mathrm{~cm}^{2} \\ \text { Finest angular resolution } & 7 \operatorname{arcsec} \\ \text { Field of view } & 2^{\circ} \\ \text { Image placement accuracy } & \sim 4 \operatorname{arcsec} \\ \text { Time resolution (statistics limited) } & \geq 0.1 \mathrm{~s}\end{array}$

\subsection{X-ray windows}

The STIX X-ray windows play two roles. First, they are a prime element in the instrument thermal control system, reflecting and reradiating most of the incident radiation, so as to limit the incident optical and IR solar flux seen by the instrument. Second, it serves to preferentially absorb the intense flux of low energy X-rays that would otherwise contribute to pulse pileup and live time issues in the detectors in large flares. By using low-Z materials, a thermallyeffective window can be developed which has acceptable X-ray absorption properties to permit observations down to $4 \mathrm{keV}$. The STIX design of the X-ray window uses optical grade beryllium. The front window at $2 \mathrm{~mm}$ thickness is screwed to 6 decoupled springs that are connected to the Solar Orbiter provided feedthrough, while the rear window at $1 \mathrm{~mm}$ thickness is directly screwed to the feedthrough (Figure 3).
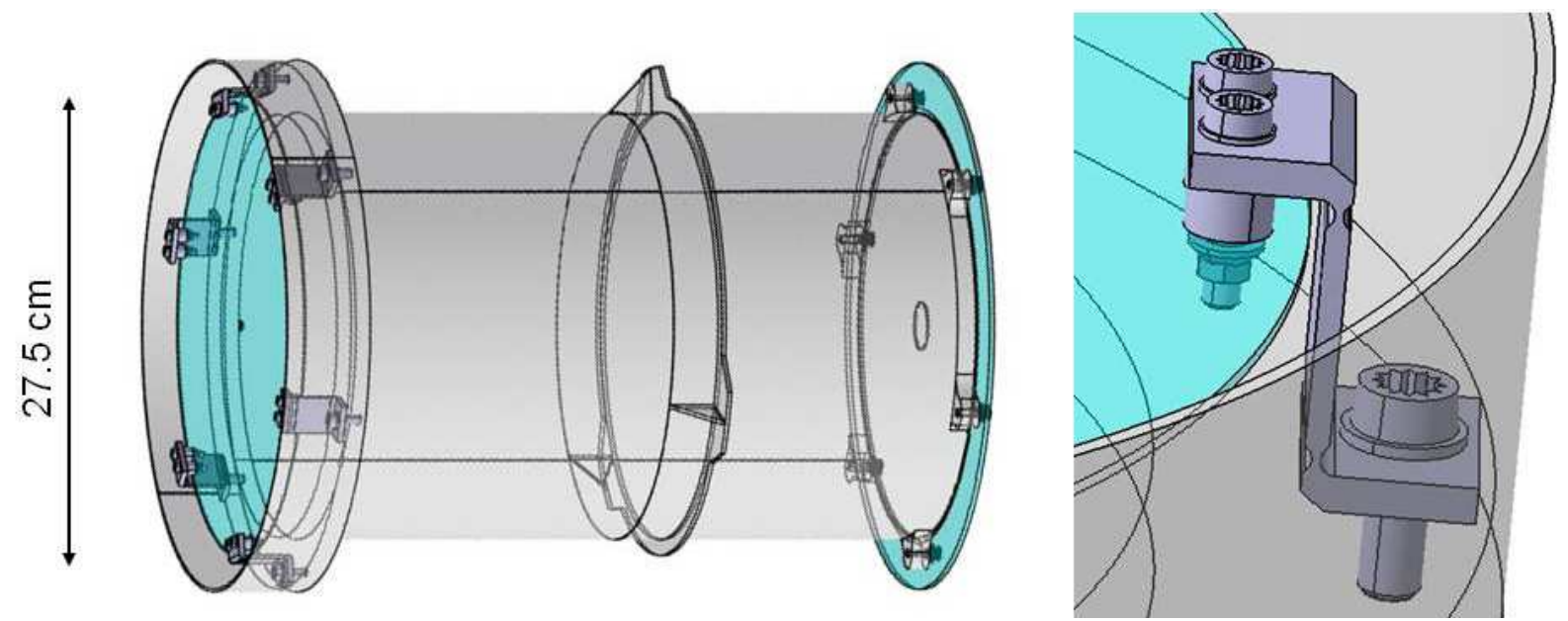

Figure 3.The STIX X-ray windows and the feedthrough. The beryllium windows are in light blue, the holes in the windows are for the aspect system.

\subsection{Imager}

Since the use of focusing optics to achieve arcsecond-class imaging over the 4-150 keV energy range is not feasible, STIX uses collimator-based, indirect imaging, similar to that used on RHESSI (but more closely related to that used by Yohkoh/HXT) to measure Fourier components of the source distribution. Details of the imaging concept are given in [3] (this volume).

Figure 4 shows the STIX Imager module. It contains two tungsten grid assemblies mounted at opposite ends of a $550 \times$ $185 \mathrm{~mm}$ diameter aluminum tube. Each grid is divided into 32 subcollimator areas each of which is located in front of a corresponding detector in the Detector Electronics Module (DEM). 
The grid areas within each subcollimator contain alternating, equispaced slits, and X-ray opaque slats (see Figure $4 \& 5$ ). For a given subcollimator, the front and rear grid slits differ slightly in pitch and/or orientation so that the transmitted Xray flux transmitted by the front and rear grids forms a large-scale Moiré pattern on the detector. A coarsely pixelated detector behind each grid pair measures the arrival time and energy of each X-ray. For a given time and energy interval, imaging information is encoded in the relative count rates of the detector's pixels. Grid parameters are chosen so that each Moiré pattern measures a specific one-dimensional spatial Fourier component of the source distribution (a visibility). Such a visibility measurement is identical to the instantaneous imaging information provided by a single antenna pair in a radio interferometer.
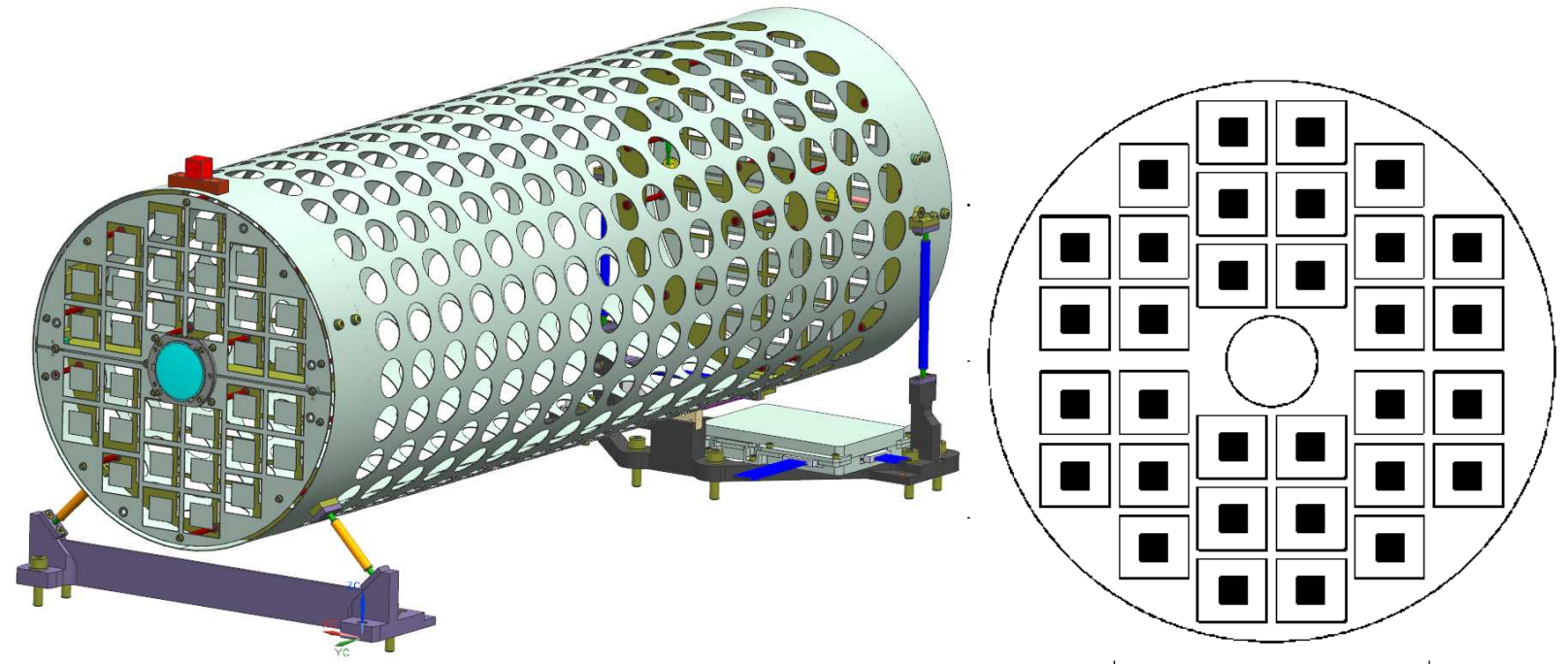

Figure 4. Left: The STIX Imager. Right: Layout of the front grid assembly. Its $180 \mathrm{~mm}$ diameter contains thirty-two $22 \mathrm{~mm}$ $\times 20 \mathrm{~mm}$ subcollimators (open rectangles) as well as room for an aspect lens at its center. The rear grid assembly is similar except that there is no lens and the subcollimators dimensions are only $15 \mathrm{~mm} \times 15 \mathrm{~mm}$. The solid squares represent the $10 \mathrm{~mm} \times 10 \mathrm{~mm}^{2}$ detectors located in the DEM behind the grids.

Thirty of the 32 subcollimators are used to measure 30 different Fourier components of the source distribution. The FWHM resolution of a subcollimator is defined as half the period of the angular response and is given by the ratio of half the grid pitch to the grid separation. The spatial frequency of the Fourier component is given by $1 /$ (twice the FWHM resolution of the subcollimator) while the orientation of the measured Fourier component is orthogonal to the direction of the slits. Grid pitches range from 0.038 to $1 \mathrm{~mm}$. For a $550 \mathrm{~mm}$ separation, this corresponds to range of angular resolutions from 7 to 180 arcseconds.

The STIX tungsten grids are fabricated with the same etch-and-stack process successfully used to make the finer RHESSI grids ${ }^{2}$. In this case up to 16 layers of etched tungsten sheets are stacked to a thickness of $0.4 \mathrm{~mm}$.

The grids are fabricated as two sets of $180 \mathrm{~mm} \times 90 \mathrm{~mm}$ semicircular segments each of which contain 16 grid elements for individual subcollimators. The semicircular grid segments are mounted on a common grid backing plate that provides mechanical support while maintaining the relative position of the two grid halves. The backing plate has a coefficient of thermal expansion (CTE) similar to tungsten so that the entire grid assembly has the same CTE.

The grids are mounted at opposite ends of an aluminum tube. The key alignment requirement is to maintain the relative twist of the two grids throughout the mission. Twist of the tube itself is prevented by bistatically mounting opposite ends at separate locations on the spacecraft panel. The front mount fixes the transverse location of the center of the tube while providing freedom to rotate about and move in the longitudinal direction. The rear mount fixes the position and orientation of that end of the tube. The cross section of the tube is maintained by circular support plates, also fabricated of aluminum, located $30 \mathrm{~mm}$ in from the opposite ends of the tube. With its cross section constrained, the tube is highly resistant to twist. The bistatic mounts prevent the introduction of torques from distortions in the spacecraft panel on which the imager is mounted. 
One of the two remaining subcollimators is dedicated to providing continuous measurements of the background and to measure the low-energy X-ray spectrum during very intense flares. The $32^{\text {nd }}$ subcollimator is configured to provide coarse but unambiguous flare location in real time.

Data processing to reconstruct the X-ray images is done after the fact on the ground using well-established computational techniques, originally developed for radio interferometry and subsequently adapted to the analysis of $\mathrm{X}$ ray data from Yohkoh/HXT and RHESSI ${ }^{4}$. STIX image quality is expected to be comparable to that obtained with Yohkoh/HXT, albeit over a wider energy range and with about six times better spectral resolution.
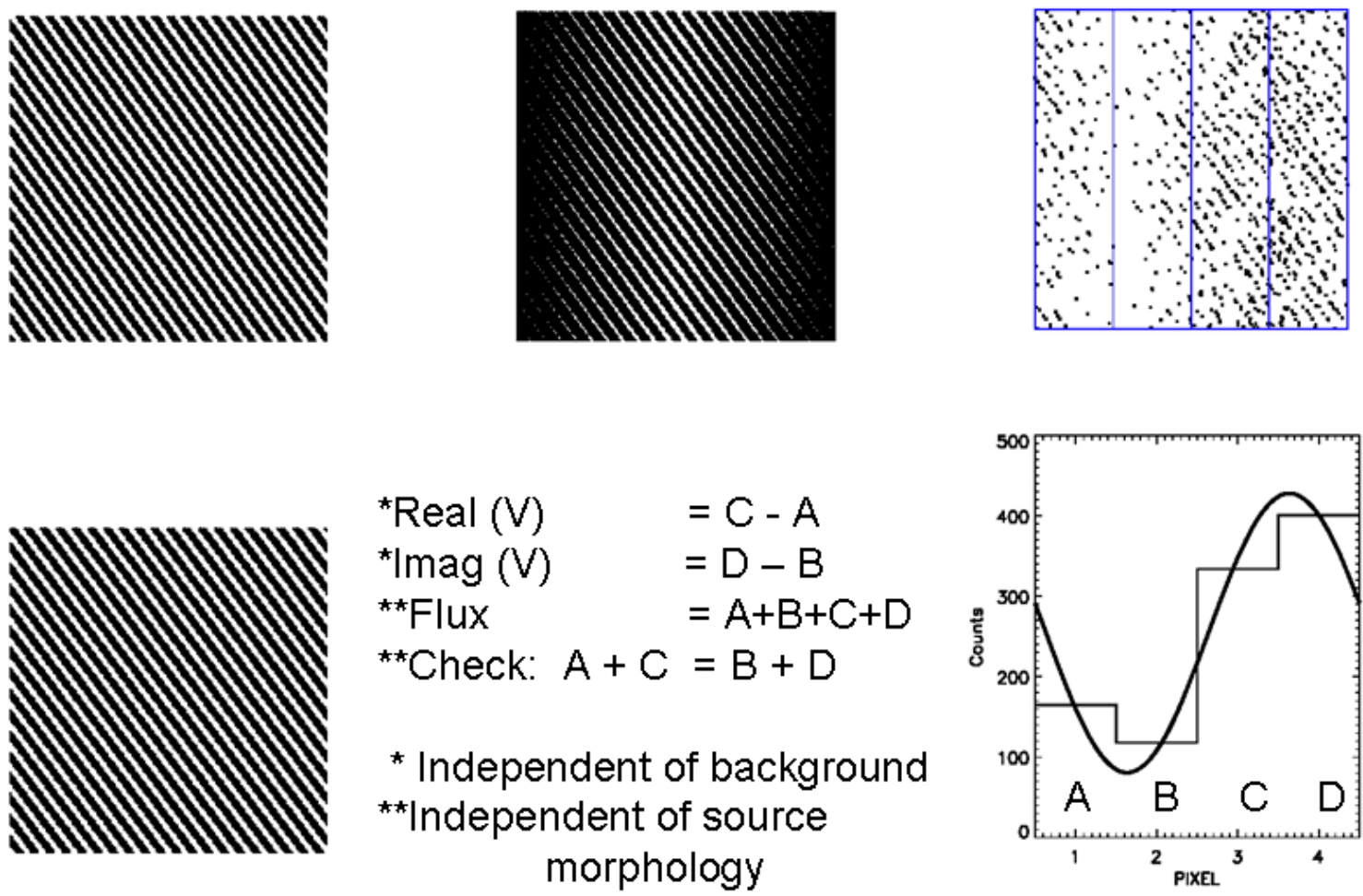

Figure 5. Measurement of visibilities. The front and rear grid elements for the subcollimator at left contain slits and slats that differ slightly in their pitch and orientation. Their combined transmission forms a Moiré pattern as illustrated at top center. The location of the maximum in this pattern depends on the source location. Simulation of a source at an arbitrarily chosen location within the field of view results in individual photons being detected at positions indicated by the dots in the upper right panel. The lower right panel shows a histogram of those locations as observed by a detector with 4 coarse pixels (blue). Sums and differences of the counts in the pixel (lower center) yield a redundant measurement of the real and imaginary components of the visibility and of the integrated flux.

\subsection{Aspect system}

The purpose of the STIX Aspect System is to allow ground analysis to reduce the pointing uncertainty with respect to the spacecraft from a guaranteed $+/-2$ arcminutes to 4 arcseconds, which is required to accurately place the hard X-ray images on the Sun. This is achieved by a lens in the front grid that produces an optical image of the solar disk on the rear grid (Figure 2). In this image plane, the grid is perforated by small apertures, arranged in a cross-shaped pattern of four „arms“ (Figure 6). The light coming through the apertures of each arm is then fed to a photodiode. Off-pointing and a changing solar image radius (due to changing distance from the Sun during the orbit) will cause the solar image to cover or uncover different apertures, leading to a modulation of the signal from each arm that can be used to calculate the locations of the solar limb, and thus determine the pointing of the telescope. 
The lens mounted in the center of the front grid is a plane-convex fused silica lens with a diameter of $3 \mathrm{~cm}$ and a focal length of $55 \mathrm{~cm}$. To reduce the amount of heat introduced into the Imager, the lens has a reflective coating that transmits the 550-700 $\mathrm{nm}$ wavelength range. The diameter of the resulting solar image on the rear grid ranges from $18 \mathrm{~mm}$ (for a perihelion of $0.284 \mathrm{AU})$ to $5.1 \mathrm{~mm}(1 \mathrm{AU})$, which is the range that has to be covered by the apertures.

Each of the four arms consists of 20 circular apertures, with diameters increasing from 90 (innermost aperture) to 320 microns. The increasing diameter ensures that each fractional signal increase resulting from an additional aperture being covered by the solar image will be larger than $20 \%$ of the total signal. This alleviates signal-to-noise issues resulting from the dark current of the photodiodes close to the Sun.

The light coming through each of the four arms is directly recorded by a UVG10 photodiode placed behind each arm on the outside of the rear grid assembly. Diodes of this type have been flown on NASA's SORCE mission. The signal produced by each diode is first amplified and then fed to a PCB board where it is digitized (12 bit) by an AD converter. All photodiodes are read out continuously, so that four time series of data are generated.

In addition to relatively large step-like signal changes which will result from the solar limb covering or uncovering a single aperture, a gradual increase between steps is caused by limb darkening (Figure 6, right). Thus the sharp steps can be used to calibrate the absolute aspect, while aspect drift can be monitored in between steps due to the gradual signal changes.
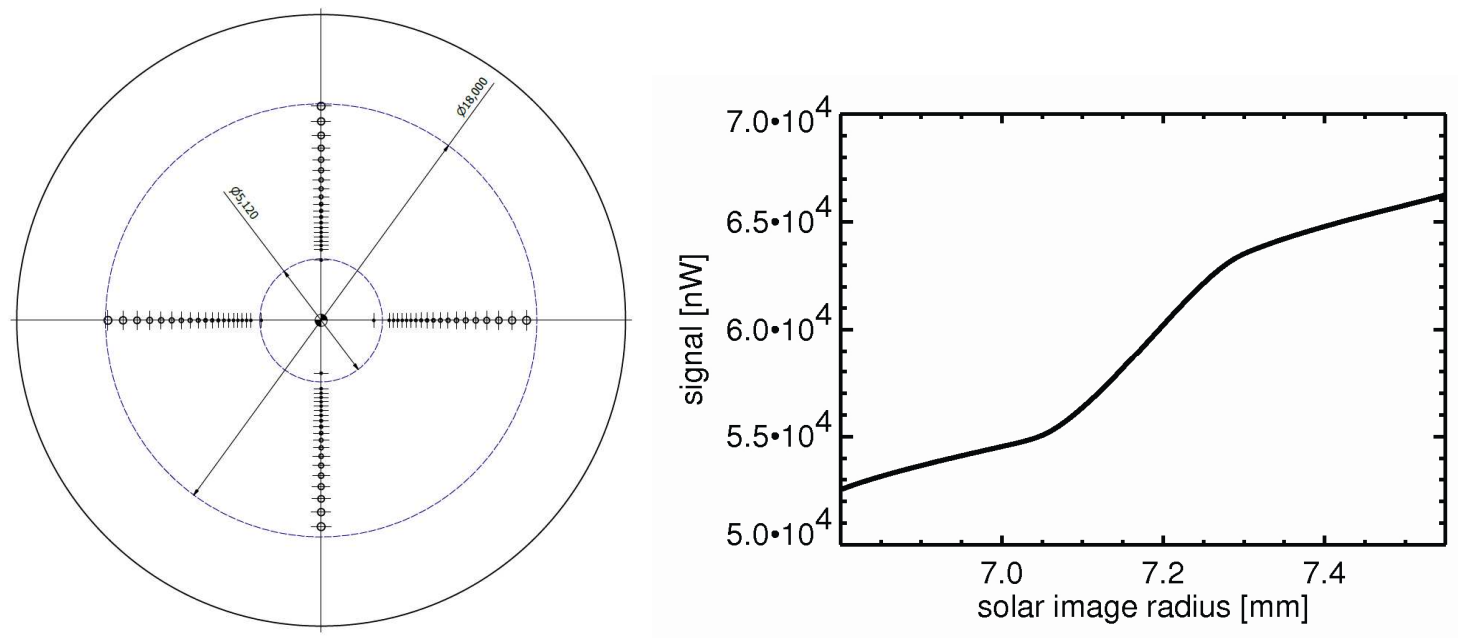

Figure 6. Left: Arrangement of apertures in the rear grid. The dashed circles represent the solar image diameter at 0.284 and 1 AU. Right: Signal increase as a function of increasing image diameter. The steep increase between 7.05 and 7.25 is due to an aperture being covered by the image, while the gradual increases are due to limb darkening.

\subsection{Detector Electronics Module (DEM)}

The DEM consists of two sub-units, the Detector Box and the Instrument Data Processing Unit (IDPU) Box (Figure 7). The DEM and Imager are mounted separately to the spacecraft. The DEM enclosure is thermally coupled to the spacecraft.

The Detector Box contains 32 structured cadmium telluride (CdTe) semiconductor crystals used for detection of X-rays, bonded to front-end hybrids called Caliste-SO. The hybrids contain an ASIC for signal amplification with selectable shaping times, for trigger generation with adjustable threshold, and for multiplexed read-out of all channels. Bias voltage of up to $1000 \mathrm{~V}$ is also routed through Caliste-SO and connected to the crystal with a thin wire glued to the cathode. The analog-to-digital converters and the digital control are located in the IDPU box.

The Caliste-SO units are mounted on front-end printed-circuit boards together with support circuitry and temperature probes. The boards are in close thermal contact with a cold plate inside the Detector Box, which in turn is connected to a spacecraft provided cold element. This arrangement is thermally isolated from the enclosure with multilayer-insulation 
foil and cools the CdTe sensors to below $-20^{\circ} \mathrm{C}$ under operating conditions (The spacecraft interior can be at temperatures of up to $+50^{\circ} \mathrm{C}$ around perihelion. See Section 2.8 for thermal design). The resulting low-leakage current is required to achieve the desired energy resolution.

Sealed Ba-133 calibration sources with a total activity well below $100 \mathrm{~Bq}$ are attached to the Detector Box entrance window for energy calibration. A weak source is required to not compromise the anticipated low-background that gives STIX its capability to detect weak solar flares.

A mechanical attenuator that is inserted autonomously by the IDPU, is mounted to the front of the Detector Box to reduce the count rate of low energy photons during intense events.

The IDPU Box contains all digital control on two redundant FPGAs, the electrical interface to the spacecraft, the lowvoltage and bias power supplies, and runs the flight software. Interface to the front-end electronics is through about 200 thin wires, signals being transmitted differentially. Minimizing the thermal bridge over the cables is important to keep the cold-element heat load low, thus their number, thickness and length has to be optimized.

The total electrical power consumption of the DEM is below $5 \mathrm{~W}$ (TBC), with $\sim 1 \mathrm{~W}$ dissipated by the front-end electronics. The weight is $3.5 \mathrm{~kg}$, the dimension $180 \mathrm{~mm} \times 200 \mathrm{~mm} \times 220 \mathrm{~mm}$.

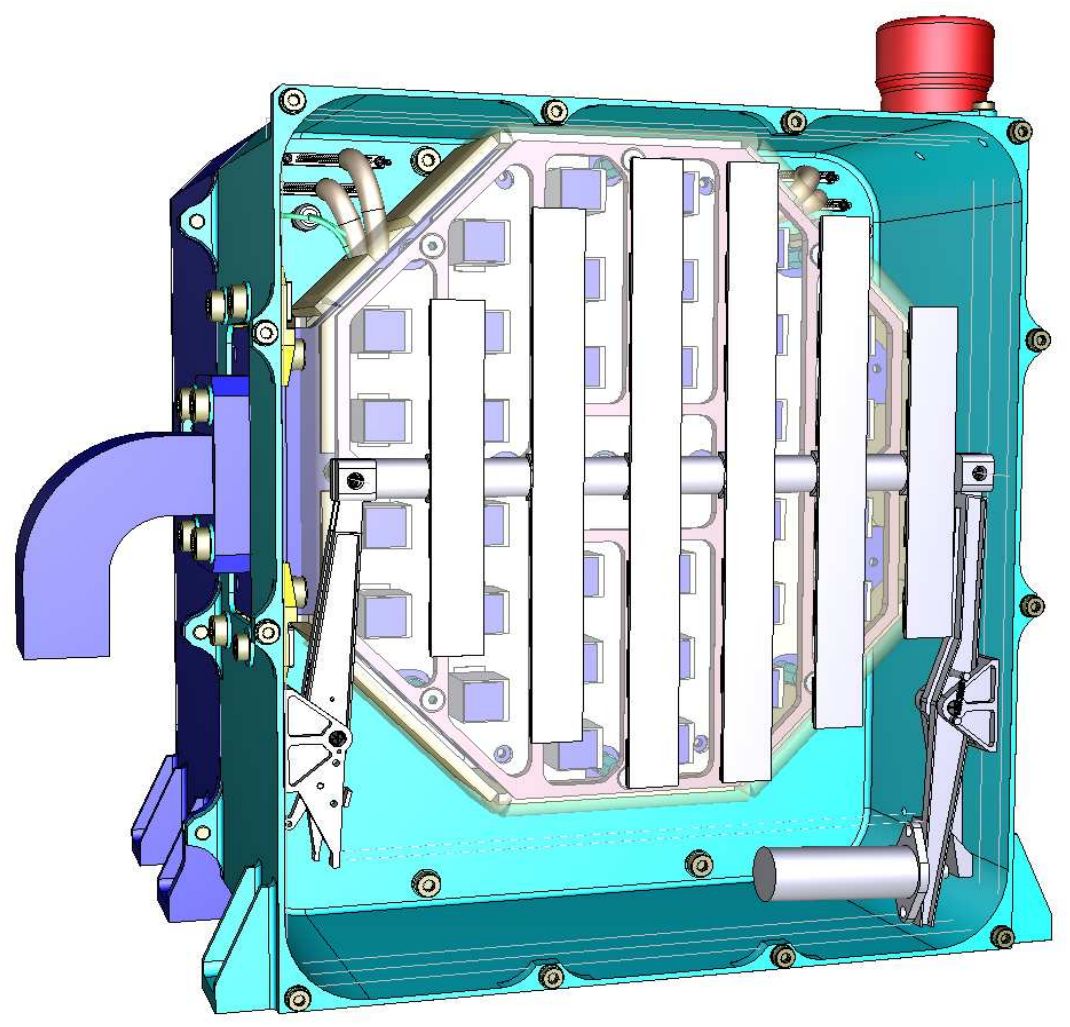

Figure 7. The Detector Electronics Module (DEM), showing the Detector Box (front-window removed for clarity), the attenuator, the thermal enclosure of the cold unit (transparent) with the space-craft cold element on the left, and the CalisteSO front-end hybrids with the CdTe crystals on top. The cables have a total length of $50 \mathrm{~cm}$ to reduce the thermal bridge from the enclosure to the cold unit and are curled up inside the thermal enclosure. The red alignment cube on top of the DEM cube will be removed before flight. The IDPU box is the rear part, with connectors to the spacecraft on its back side.

\subsection{CdTe sensors}

CdTe sensors are purchased from Acrorad Co., Ltd., Japan. The detectors dedicated for STIX are $10 \times 10 \times 1 \mathrm{~mm}^{3}$ in size. The electrical contacts are fabricated by Acrorad using two different metal deposition techniques. The cathode consists of electroplated platinum. The anode is metal evaporated and composed of an Al/Ti/Au multilayer (Schottky 
contact). As-purchased, this layer is not patterned apart from a $50 \mu \mathrm{m}$ wide electrode free area surrounding the anode side (dicing gap). The electrode-free zone improves the quality of the detector edges and leads later on to a damage free guard ring.

One CdTe sensor consists of four identical side-by-side areas (stripes), each stripe consisting of 2 large pixels and 1 small pixel, giving a total of 12 pixels on each chip (Figure 8, left). The 8 large pixels and 4 small pixels are arranged in an asymmetrical geometrical configuration surrounded by a guard ring. The pixel effective areas range from $9.7 \mathrm{~mm}^{2}$ for the big and $1.0 \mathrm{~mm}^{2}$ for the small ones. A $50 \mu \mathrm{m}$ wide electrode-free zone separates each adjacent pixel and the guard ring and pixel (see Figure 8, left). The small pixels are used to reduce the effective area when large X-ray fluxes would cause excessive dead time or pulse pileup ${ }^{3}$.

In order to create pixels and a guard ring out of the $\mathrm{Al} / \mathrm{Ti} / \mathrm{Au}$ multilayer a segmentation process was developed at the Paul Scherrer Institute (PSI). The process is based on reactive ion etching with fluorine etching gases. A photo-resist pattern serves as etching mask in order to transfer the pixel layout into the electrode. Before patterning the anode side, all detectors are I-V measured and visually inspected. For the I-V measurement a bias voltage of up to $1000 \mathrm{~V}$ is applied. Best detectors show leakage currents below $15 \mathrm{nA}$ at $1000 \mathrm{~V}$ (electrode size $10 \times 10 \mathrm{~mm}^{2}$ ) at room temperature.

For the selection of flight-quality detectors two setups are under development. One setup allows serial measurement of the leakage current of each individual pixel at different temperature levels. Leakage current determines energy resolution and is therefore one of the key parameters. The second setup was designed to perform spectral measurements with different radioactive sources to determine energy resolution.

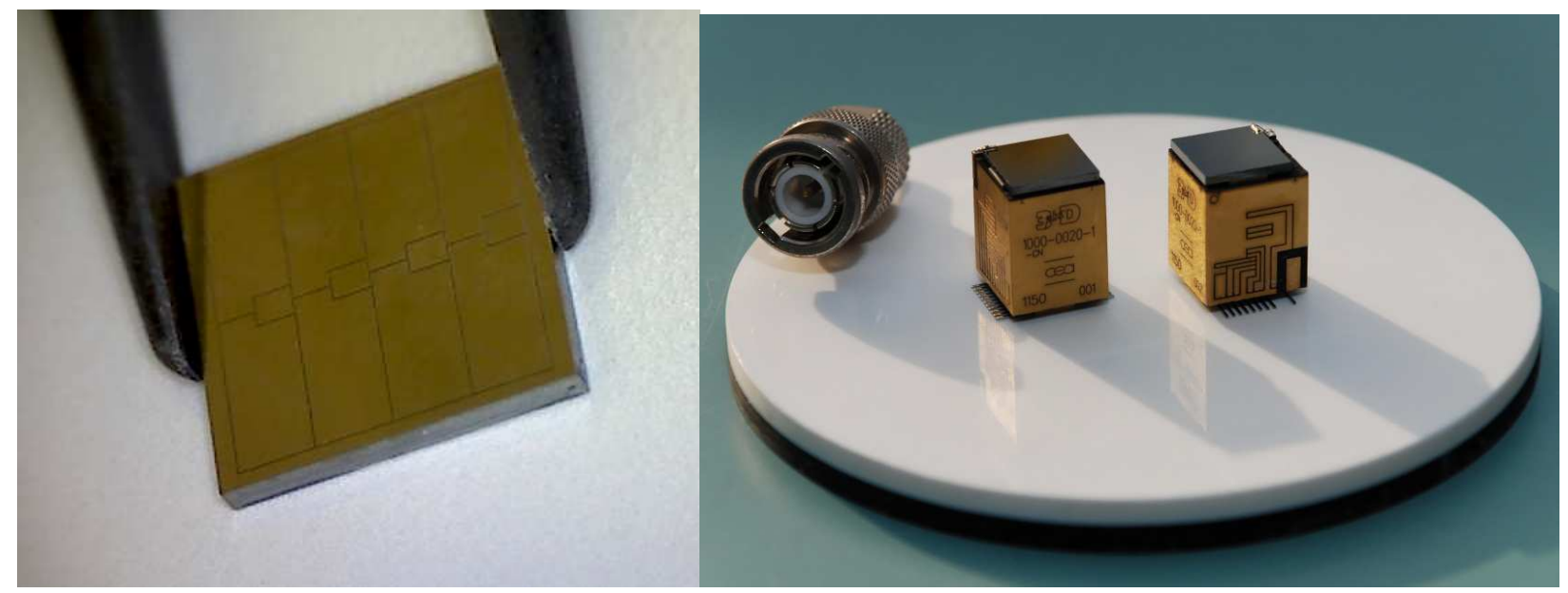

Figure 8. Left: Close-up view of the segmented anode side of a CdTe detector $10 \times 10 \times 1 \mathrm{~mm}^{3}$ in size held in a pair of tweezers. Eight large and four small pixels are surrounded by a guard ring in order to reduce the leakage current in the pixels. Right: First two prototypes of Caliste-SO spectrometer units. The device is $17 \mathrm{~mm}$ high and includes a $1 \mathrm{~mm}$-thick CdTe pixel sensor (as shown on the left), a low noise analog front-end ASIC called IDeF-X HD and a 20-pin electrical interface at the bottom for a surface mounting on the STIX front-end board. The electrical body is slightly longer than the crystal to get a pad at the top for the high voltage wire bonding to the detector cathode.

\subsection{Caliste}

The detection unit of STIX is called Caliste-SO; it is the Solar Orbiter version of a concept of hard X-ray micro-camera developed by CEA, 3D Plus, and CNES for high energy astrophysics ${ }^{5}$. The device integrates the functions of X-ray absorption with a CdTe semiconductor detector and analog front-end electronics with a full custom ASIC named IDeF-X $\mathrm{HD}^{6}$; Figure 8 (right) shows the resulting hybrid component with a CdTe crystal at the top and a small outline package (SOP) at the bottom as an electrical interface. The strong advantages of this design for a small instrument on-board a mission to the Sun are the compact volume $\left(12 \times 14 \times 17 \mathrm{~mm}^{3}\right)$, the low mass $(5 \mathrm{~g})$, and the low power consumption $(20$ $\mathrm{mW}$ for 12 pixels). Moreover, the ASIC was proved to be radiation hard (latch-up free up to $110 \mathrm{MeV} \mathrm{cm}^{2} \mathrm{mg}^{-1}$, working after $250 \mathrm{krad}$ total ionizing dose). 
Four prototypes were developed and characterized during the Solar Orbiter implementation phase. For each device, four circuits were fabricated: one with the IDeF-X HD ASIC, two with passive parts for the power supply filtering and the local decoupling of reference voltages and one for the routing of the high voltage from the bottom interface to the detector cathode. The circuits were stacked perpendicular to the detection surface and molded into an epoxy resin according the 3D Plus technology ${ }^{7}$. The 20-pin SOP interface was added at the bottom and the top surface was prepared to receive the pixel detector; the resulting part is the so-called Caliste-SO electrical body. Electrical characterizations were performed before receiving crystals. Charges coming from the semiconductor detector after photon interaction are simulated using the injection test input. Dark current flowing in the sensor during operation can be reproduced using a programmable current source integrated in the ASIC. Noise performance is close to identical from one device to another. With a $20 \mathrm{pA}$ leakage current per channel, equivalent noise charge was measured to be 58 electrons rms at $6 \mu$ s peaking time (optimal value) and 74 electrons rms at $1.4 \mu$ s (shortest shaping for high count rate operation in STIX). The CdTe pixel sensors from PSI were then mounted using a laser-assisted polymer bump bonding technique ${ }^{8}$. Two gold wires were bonded to connect the high voltage from the pad at the electrical body surface to the CdTe cathode.
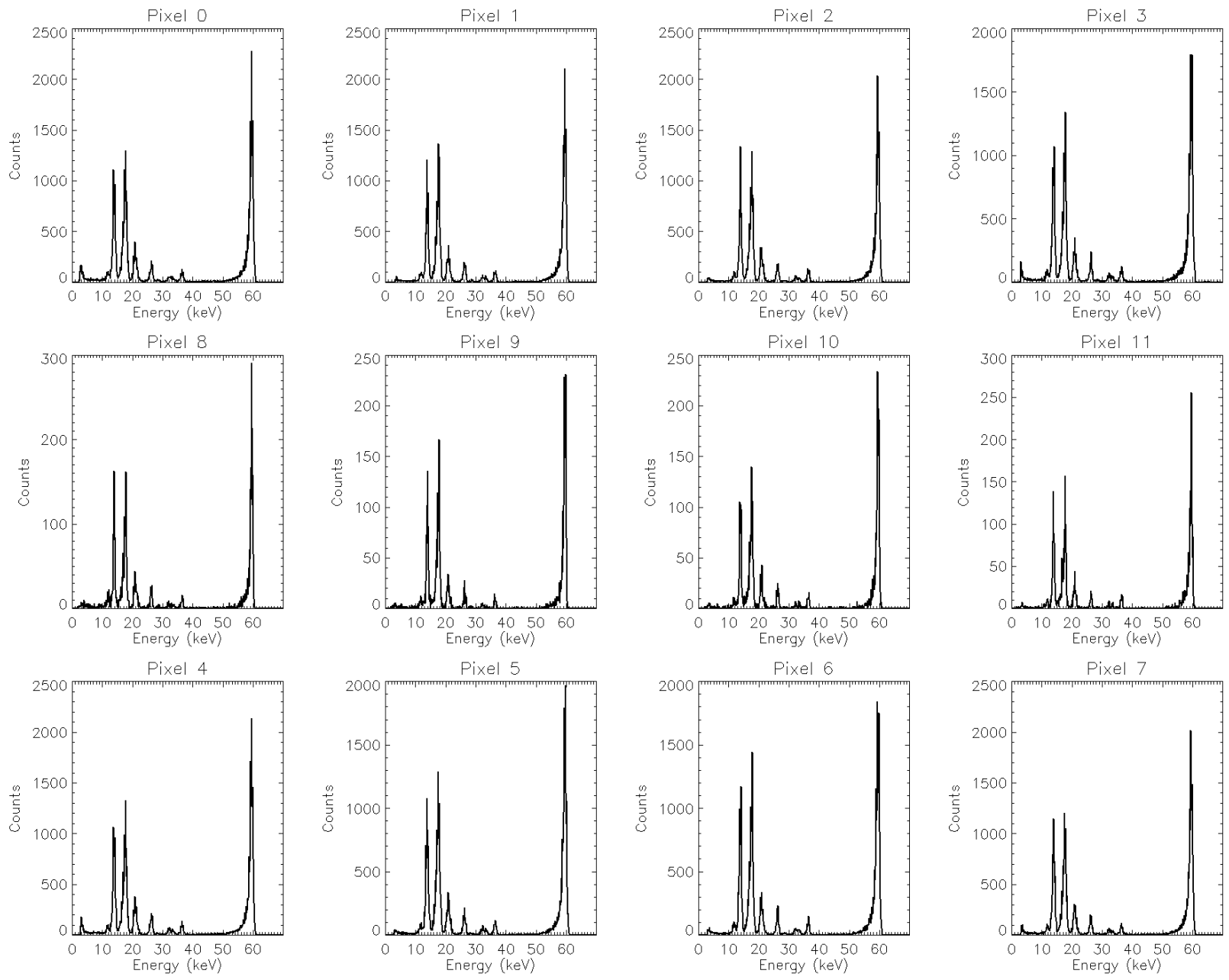

Figure 9. Caliste-SO first light: ${ }^{241} \mathrm{Am}$ spectra recorded on each pixel of one Caliste-SO prototype at $-30^{\circ} \mathrm{C}$. The detector is biased at $-200 \mathrm{~V}$, the peaking time of the ASIC is set to $1.4 \mu$ s. Pixels $\# 0$ to $\# 7$ are large pixels $\left(9.7 \mathrm{~mm}^{2}\right)$ and show an energy resolution of $1.3 \mathrm{keV} \mathrm{FWHM}$ at $14 \mathrm{keV}$ and a low-level threshold around $3.4 \mathrm{keV}$. Pixels \#8 to \#11 are small pixels in the center $\left(1 \mathrm{~mm}^{2}\right)$ and show an energy resolution of $0.9 \mathrm{keV} \mathrm{FWHM}$ at $14 \mathrm{keV}$ and a threshold as low as $2.4 \mathrm{keV}$. 
Spectroscopic measurements were performed for one Caliste-SO prototype using a 241 Americium source. The device was inserted in a test socket and placed in a vacuum chamber. A cold finger was in contact with 2 lateral sides to efficiently cool the micro-camera down to $-30^{\circ} \mathrm{C}$. (The flight Caliste-SO devices will be equipped with copper thermal drains between the pins to evacuate the dissipated power from the bottom to the cold plate.) Spectra were recorded in the 12 pixels (see Figure 9) and individual calibration was performed. To derive the spectral resolution, spectra were built by separately summing the counts in the 4 small pixels and in the 8 large pixels. The small pixels provide energy resolutions of $0.94 \mathrm{keV}$ at $14 \mathrm{keV}$ and $1.14 \mathrm{keV}$ at $60 \mathrm{keV}$, while the large pixels give $1.31 \mathrm{keV}$ at $14 \mathrm{keV}$ and $1.52 \mathrm{keV}$ at $60 \mathrm{keV}$ for the large pixels. The low-energy threshold can be set as low as $2.4 \mathrm{keV}$ for the small pixels and $3.4 \mathrm{keV}$ for the large ones. The best performances are obtained with the small pixels because dark current and input capacitance — the 2 main contributions to electronic noise - are proportional to the electrode surface.

\subsection{Instrument Data Processing Unit (IDPU)}

The Instrument Data Processing Unit (IDPU) includes CPU microprocessors, memories for data accumulation and software. It interfaces to the Payload Data Management Unit, a housekeeping system and other STIX electronics. A combination of low level state machines and high level software allows the IDPU to control and acquire data from Caliste units, make readouts from diodes in the Aspect system (see section 2.3), manage the memories and control the attenuator and the power supply units (PSU).

The central element of the IDPU is a Field Programmable Gate Array (FPGA) from Actel's RTAX S/SL family (Figure 10). The FPGA is connected to a Programmable Read Only Memory (PROM), a Random Access Memory (RAM) and a Flash memory. The PROM serves as a storage element for Start-Up software and is used only during the start-up of the system. The RAM of $128 \mathrm{MB}$ size has double functionality. While half of it is used as an operation memory for the application software, the other half serves as a rotating buffer for data coming from the detectors. The Flash memory with capacity of $16 \mathrm{~GB}$ functions as an archive memory that allows us to store several months of scientific data. Additionally, the Flash memory contains two copies of the application software, which is used during the instrument operation. The microprocessor used in the IDPU is Leon 3FT from Aeroflex and is loaded into the FPGA. The processor is extended by an error detection and correction intellectual property (EDAC IP) core. The EDAC allows correcting one bit-error and detecting two bit-errors in 32-bit word at a time. Memory scrubbing for archive memory is employed as well.

Internal housekeeping (HK) of the instrument is a part of the IDPU. It consists of four analog to digital converters and additional analogue circuitry.

The IDPU consists of two functionally and electrically identical printed circuit boards (PCB): main and redundant. It communicates with the spacecraft via SpaceWire. The SpaceWire interface makes use of a controller implemented in FPGA and external physical-layer transceivers. The SpaceWire can be used to communicate with the spacecraft via two links, but only one of them is active at a time. The SpaceWire is cross-coupled to both IDPU PCBs and thus operates in warm redundancy concept. This means that if one link fails there is no need to switch the IDPU to redundant unit. The IDPU itself operates in cold redundancy concept. It means that if a malfunction of the IDPU component occurs, the whole unit has to be switched to the redundant PCB. Since each of the IDPU PCB is supplied from the PSU independently without cross-coupling, the switching to the redundant PCB is done by the spacecraft by switching to the redundant PSU.

The software (S/W) implemented in the IDPU consists of three components: the Start-up S/W, Application S/W and Basic S/W. The Start-up is boot software that is always started right after the instrument is powered on. It checks the critical subsystems in the STIX instrument and after that it executes the Application S/W. The Application S/W, as its name suggests, is the main application used when the STIX instrument is active. It works on the RTEMS operating system and performs high level operations such as: data compression, real-time data analysis for detecting and signalizing the flare to the $\mathrm{s} / \mathrm{c}$ for optional transmission to other instruments, telecommand and telemetry services, high level control of STIX subsystems. The Basic S/W is an independent library that provides the drivers to access the hardware. It is used by both Start-up and Application software. 


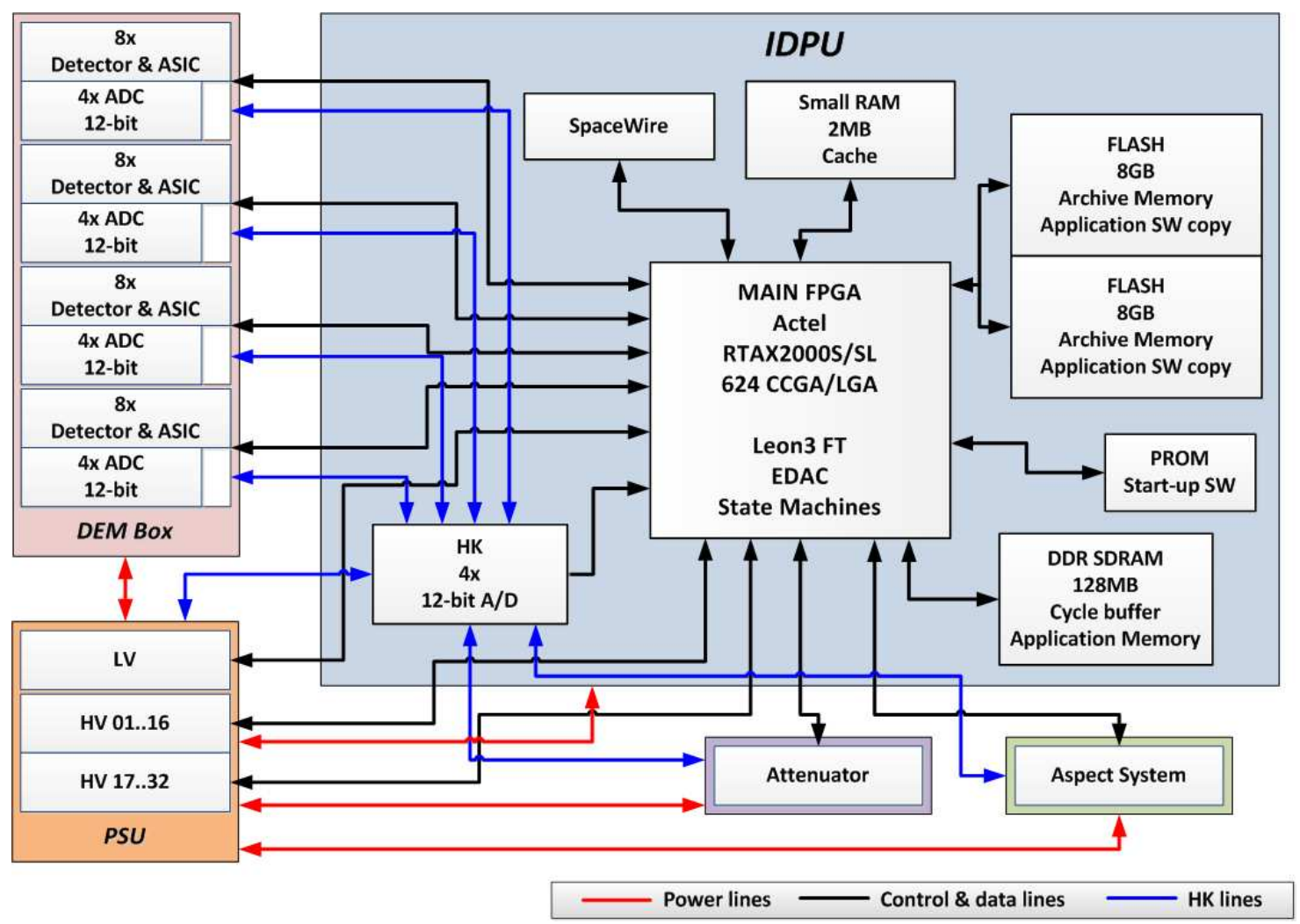

Figure 10. The STIX data flow: The X-rays are detected by Caliste-SO detectors which generate triggers. These triggers are forwarded by ASIC electronic through LVDS lines to the IDPU. The state machines detect the triggers in the FPGA. After the detection, a series of control signals are sent to ASICs and A/D converters associated with them. These cause the conversion of the analog voltage levels, that are proportional to X-ray energy, to 12-bit digital values. These are then converted to 32 non-linear detector-matched energy channels for subsequent use. The counts are accumulated using variable duration accumulators, compacted and stored in the archive memory for subsequent data selection and compression. The memory is scaled to contain several months of data. The energy and time resolution of the archived data is such that no statistically or spectrally significant information has been lost. Data selection and compression algorithms are based on the on-board flare detection and adaptive algorithms, supplemented by specific requests from the ground. Other data processing functions include: quick-look data accumulations; live-time measurement; background monitoring; on-board coarse flare location; acquisition and compression of aspect data; and the quiet-time long-term accumulation of background counts with 10-bit energy resolution to monitor the detector energy calibration.

\subsection{Thermal design}

The STIX thermal design is driven by the following requirements:

1. The X-ray windows should be partially transparent down to $4 \mathrm{keV}$.

2. To avoid distortion of the grids by temperature gradients, the temperature gradient across the grid should be smaller than $30^{\circ} \mathrm{C} / \mathrm{m}$ (which is equivalent to $\Delta \mathrm{T}<6^{\circ} \mathrm{C}$ between any points on grids).

3. The operational temperature of the detectors should be in the range of $-50^{\circ} \mathrm{C}$ to $-20^{\circ} \mathrm{C}$ (at higher temperature the spectral resolution of the detectors decreases rapidly).

4. The electronics operational temperature should be in the range of $-30^{\circ} \mathrm{C}$ to $+60^{\circ} \mathrm{C}$; the temperature of the Imager should be below $100^{\circ} \mathrm{C}$. Furthermore the temperature of the subsystems should not exceed the temperature limits in the non-operational phase. 
The STIX thermal design encounters the following challenges: (i) it should limit the incoming heat flux in the visible and infrared range by using appropriate window characteristics, (ii) it should stabilize the temperature of the Imager by reducing the conduction heat flow to the spacecraft, and (iii) it should separate the detector box into two separated heat circuits (cold detectors and hot detector box). The scheme of the design is shown on Figure 11.

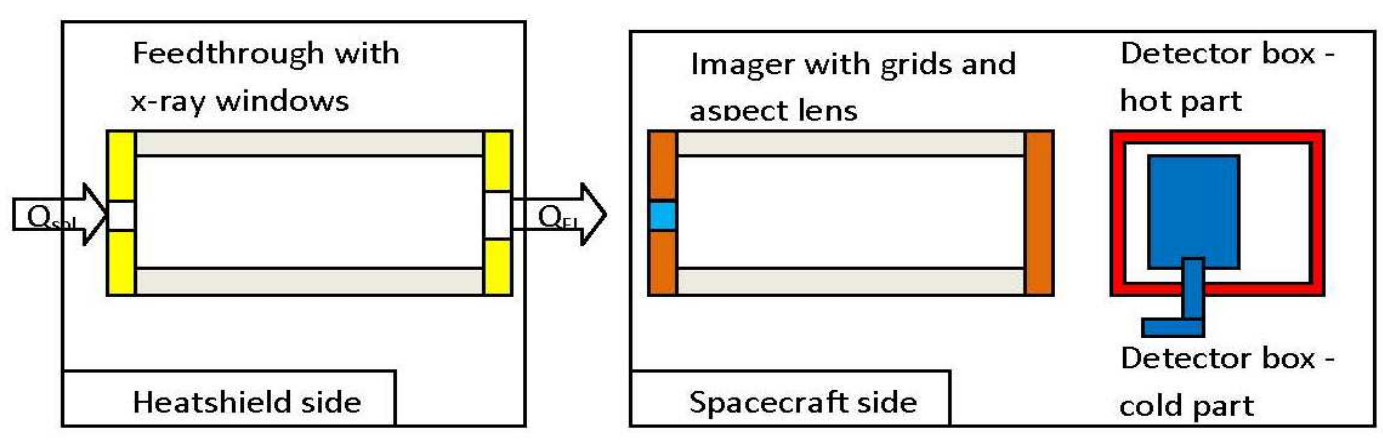

Figure 11.The scheme of the STIX instrument thermal design. $\mathrm{Q}_{\text {sol }}$ is the solar heat flux equal to $17.6 \mathrm{~kW} / \mathrm{m}^{2}$ at $0.28 \mathrm{AU}, \mathrm{Q}_{\mathrm{FI}}$ is the heat flux between rear X-ray window and the Imager.

In space applications the heat is exchanged only in a conductive and radiative way. Therefore, it requires solving the Fourier equation with a linear term describing the conductive flow and a nonlinear $\left(\sim \mathrm{T}^{4}\right)$ term describing the radiative exchange. To solve the nonlinear partial differential equation the model was approximated by dividing the geometry into the elements and calculating between all of them the conductive and radiative terms. For the radiative terms, the Monte Carlo approach was used to calculate of the incoming and outgoing fluxes, which is especially important for specular surfaces.

The most demanding case to model is the closest approach to the Sun with the following parameters:

- Solar heat flux of about $17.6 \mathrm{~W} / \mathrm{m}^{2}$ in visible range,

- Feed-through temperature is $175^{\circ} \mathrm{C}$ at the bottom and $270^{\circ} \mathrm{C}$ at the top,

- Spacecraft temperature at $50^{\circ} \mathrm{C}$,

- Cold element temperature at $-25^{\circ} \mathrm{C}$,

- Conductance between detector box and S/C of about $6 \mathrm{~W} / \mathrm{K}$.

Within the detector electronics module, the front-end electronics generate $0.8 \mathrm{~W}$ and the IDPU electronics up to $4 \mathrm{~W}$ (TBC).

The current design includes the following set of materials to fulfill the requirements:

- Beryllium windows with an appropriate top coating to minimize the heat flow to the Imager to $<0.7 \mathrm{~W}$;

- Imager mounting is designed to minimize the conductive heat flow (less than $0.0056 \mathrm{~W} / \mathrm{K}$ );

- The imager is painted black to an equally distributed temperature below $100^{\circ} \mathrm{C}$;

- Reflective layer $(>80 \%)$ on the Aspect lens limits the direct Sun flow to the imager;

- Insulation between the cold and hot subsystems of the DEM minimizes the conduction to $0.9 \mathrm{~W}$ and the radiation to $0.27 \mathrm{~W}$;

- Light and high conductive Al-Be alloy was used to keep the detector temperature below $-20^{\circ} \mathrm{C}$. The thermal resistance of this part is limited to $0.85 \mathrm{~K} / \mathrm{W}$.

The resulting temperatures in the instrument subsystems are presented in Figure 12. 


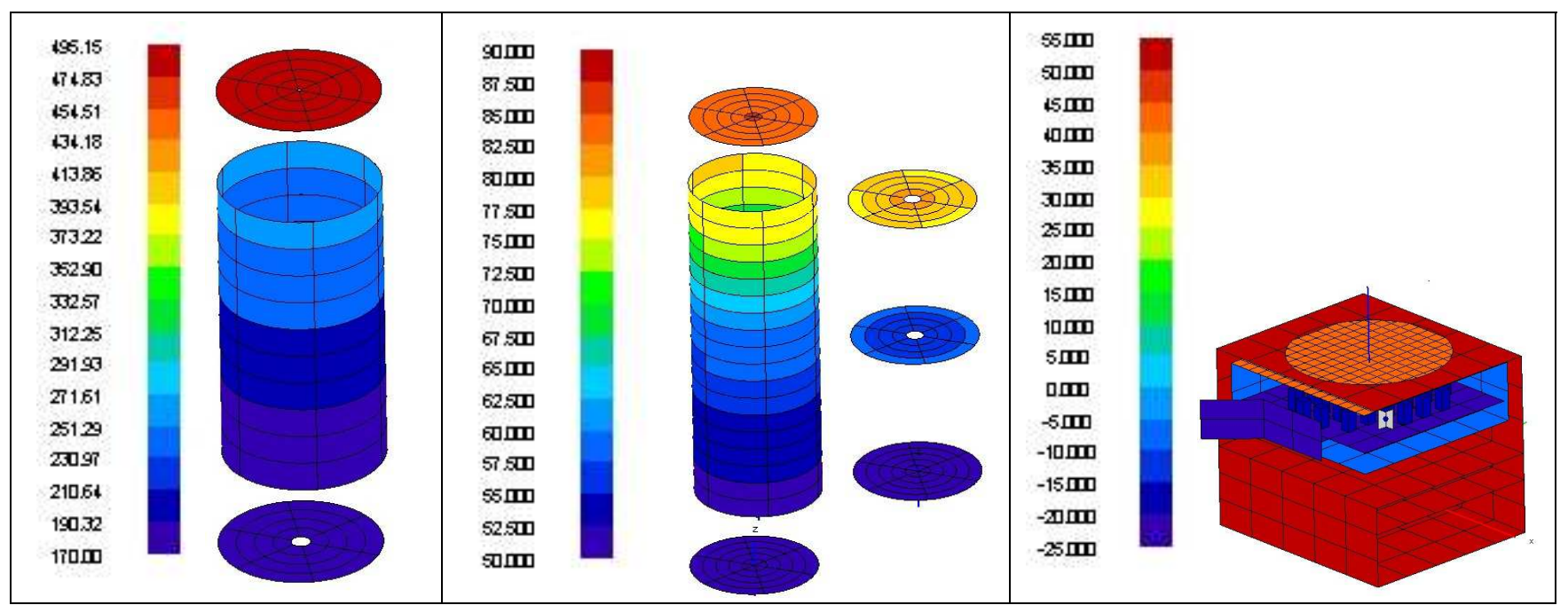

Figure 12. The temperature profiles of the STIX instrument in the hot operational case. On the left the Feedthrough submodel with the X-ray windows is presented. In the middle the Imager sub-model with the aspect lens, the grids and its supports is shown. On the right the Detectors Electronics Module is presented.

\section{SUMMARY}

STIX is a hard X-ray spectrometer and imager operating in the $4-150 \mathrm{keV}$ range. Imaging is achieved by 32 pairs of absorbing tungsten grids, sampling different spatial Fourier components. This imaging concept was used in previous missions and is here significantly improved. An important novelty in STIX is the pixelization of the sensor, making it possible to measure a Fourier component with just one subcollimator. Additionally, the measurement is independent of the background count rate. The front-end hybrid Caliste uses a versatile ASIC and technologies developed previously for a non-solar mission ${ }^{4,5}$. The design of Imager and IDPU sub-units reduced the STIX instrument in mass and power consumption by an order of magnitude over previous instruments, yet it provides a powerful contribution to the Solar Orbiter suite of instruments exploring the dynamics of solar inputs to the heliosphere.

The modularity of the STIX concept makes it attractive for use in future solar hard X-ray instruments. The design could be easily reduced to a single detector unit working as a simple flare locator. On the other hand, a larger number of subcollimators would provide enhanced image quality and better sensitivity. Using thicker grids and sensors, the STIX detector concept could be extended to image higher photon energies. For gamma-ray imaging a longer Imager is required to make the aspect ratio of the grids feasible ${ }^{9}$.

The relatively easy reproducibility of STIX concurs with the desire for a second STIX instrument operating simultaneously. A STIX-like instrument at another position in the solar system or in earth orbit would open the possibility of stereoscopic hard X-ray observations. Comparison of such inter-calibrated observations would permit direct observation of the directivity of hard X-ray emission as a function of position and so determine the extent of beaming of the corresponding non-thermal electrons. Such observations would also allow us to locate hard X-ray sources in three dimensions and to determine the volume of hard X-ray sources. Furthermore, by investigating events that are seen partially disk-occulted by one instrument, but seen on-disk by the other one, the relation between the footpoint sources relative to the much fainter coronal sources could be systematically studied for the first time. This would allow us to directly measure the electron distribution in the coronal acceleration site and investigate how electrons escape the acceleration site to impact on the solar surface and how electrons escape into the heliosphere. 


\section{REFERENCES}

[1] Kosugi et al., "The Hard X-ray Telescope (HXT) for the SOLAR-A Mission", Solar Physics 136 (1991) 17.

[2] Lin et al., "The Reuven Ramaty High-Energy Solar Spectroscopic Imager (RHESSI)", Solar Physics 210 (2002) 3.

[3] Hurford G., et al., "The imaging concept for the spectrometer/telescope for imaging X-rays (STIX) on Solar Orbiter", Proc. SPIE 8443 (2012) 8443-130.

[4] Hurford et al.,"The RHESSI Imaging Concept", Solar Physics 136 (2002) 61.

[5] Limousin O. et al., "Caliste 256: A CdTe imaging spectrometer for space science with a $580 \mu \mathrm{m}$ pixel pitch", Nucl. Instrum. and Meth. A 647 (2011) 46-54.

[6] Michalowska A. et al., "IDeF-X HD: A low power multi-gain CMOS ASIC for the readout of Cd(Zn)Te detectors", Nucl. Sci. Symp. Conf. Rec. (2010) 1556-1559.

[7] Val C., Leroy M., Patent 90 15473, 3D Plus (1990).

[8] Limousin O., Soufflet F., Procédé d'interconnexion par retournement d'un composant électronique, Institut National de la Propriété Intellectuelle, Patent INPI/00719 (2011).

[9] Matthews et al., "Solar Particle Acceleration Radiation and Kinetics (SPARK). A mission to understand the nature of particle acceleration", Experimental Astronomy 33 (2012) 237. 